\title{
VISCERAL PERCEPTION
}

\section{Visceral perception: inflammatory and non-inflammatory mediators}

\section{Bueno, J Fioramonti}

Gut 2002;51 (Suppl I):i19-i23

Visceral hypersensitivity is currently the most widely accepted mechanism responsible for abdominal pain. Inflammatory mediators are known to sensitise primary afferents and to recruit silent nociceptors. Recent evidence suggests that non-inflammatory mediators also have the potential to trigger visceral pain. This sequence of events may constitute part of an alerting system which prompts the central nervous system to correct gastrointestinal responses to ingestion.

\begin{abstract}
SUMMARY
Visceral hypersensitivity is currently the most widely accepted mechanism responsible for abdominal pain and contributes to intestinal motor abnormalities in functional gastrointestinal disorders such as irritable bowel syndrome (IBS). Inflammatory mediators are known to sensitise primary afferents, especially $\mathrm{C}$ fibre polymodal receptors, and to recruit silent nociceptors. Local tissue injury results in the release of a variety of chemical substances (for example, potassium, adenosine triphosphate (ATP), bradykinin, and prostaglandin $\mathrm{E}_{2}\left(\mathrm{PGE}_{2}\right)$ ) which directly activate nerve endings and trigger the release of algesic mediators directly from immunocytes and mast cells. At the same time, other sensory neuropeptides released by axon reflexes activate neutrophils, fibroblasts, and mast cells resulting in the so called "neurogenic phase" of inflammation. Recent evidence suggests that noninflammatory mediators also have the potential to trigger visceral pain or to lower the threshold of neuronal responses to mechanical stimuli. Stress, for example, affects visceral sensitivity without triggering an intestinal immune response. Chemicals such as glycerol sensitise primary afferent neurones; peripherally released glutamate and trypsin also have direct actions on nociceptive fibres. This sequence of events may constitute part of an alerting system which prompts the central nervous system (CNS) to correct gastrointestinal responses to ingestion.
\end{abstract}

See end of article for authors' affiliations

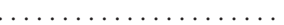

Correspondence to: Dr L Bueno, Department of Pharmacology INRA, 180

Chemin de Tournefeuille, BP3, 31931 Toulouse Cedex 9, France;

lbueno@toulouse.inra.fr

\section{INFLAMMATORY MEDIATORS OF VISCERAL PERCEPTION \\ Origin and nature of inflammatory mediators}

Local tissue injury prompts the release of chemical mediators (potassium, hydrogen ions, ATP, and bradykinin) and inflammatory mediators (for example, $\mathrm{PGE}_{2}$ ) from inflammatory cells. These substances directly activate nerve endings and trigger the release of algesic mediators (for example, histamine, serotonin (5-HT), nerve growth factor (NGF), and prostanoids) from other cells and afferent nerves. ${ }^{1-3}$ This sensitises the endings of afferent nerve terminals resulting in an increased response to painful stimuli.

Prostaglandins and other arachidonic acid derivatives increase the sensitivity of nerve terminals to bradykinin and other pain producing substances. A cascade of events involving substance P (SP), histamine, 5-HT, and cytokines in secondary neurones causes nearby nociceptors to become sensitised. The close proximity between mast cells and the endings of sensory neurones results in an amplifying loop in which SP released from nerve endings activates mast cell degranulation, releasing histamine. This induces further release of SP from sensory nerve endings and also triggers the release of NGF which further exacerbates the situation by promoting the development and enhancing the function of sensory neurones. ${ }^{3}$

Although mast cells play a major role in the sensitisation of primary afferents, there is increasing evidence that substances released from other local entities are of additional importance (fig 1). In the presence of inflammation, prostaglandins released from macrophages and sympathetic terminals are thought to have a direct action on receptors located on afferent endings. ATP is released from sympathetic nerves which innervate visceral smooth muscle. It acts as a cotransmitter for noradrenaline and is thought to bind to the P2X3 subtype of purine receptors located on terminal endings thereby generating pain signals. ${ }^{4}$ Eicosanoids such as $8(\mathrm{R}), 15(\mathrm{~S})$ dihydroxyeicosatetraenoic acid released from neutrophils directly affect adenyl cyclase activity in afferent neurones, and it is likely that cytokines secreted by the macrophages indirectly affect neuronal activity. ${ }^{2}$

This cascade of events places the primary afferents in a state of permanent sensitisation resulting either from increased concentrations of bradykinin, 5-HT, eicosanoids, or ATP at specific terminal neurone receptors, or as a result of interleukin (IL) release from immunocytes. Activation of local adrenergic fibres may also sensitise primary afferent endings (fig 1). This activated

Abbreviations: ATP, adenosine triphosphate; CGRP, calcitonin gene related peptide; CNS, central nervous system; IBS, irritable bowel syndrome; IL, interleukin; NGF, nerve growth factor; NKA, neurokinin $A ; P_{2}$, prostaglandin $\mathrm{E}_{2} ; \mathrm{SP}$, substance $\mathrm{P}$; TNF, tumour necrosis factor; 5-HT, serotonin. 


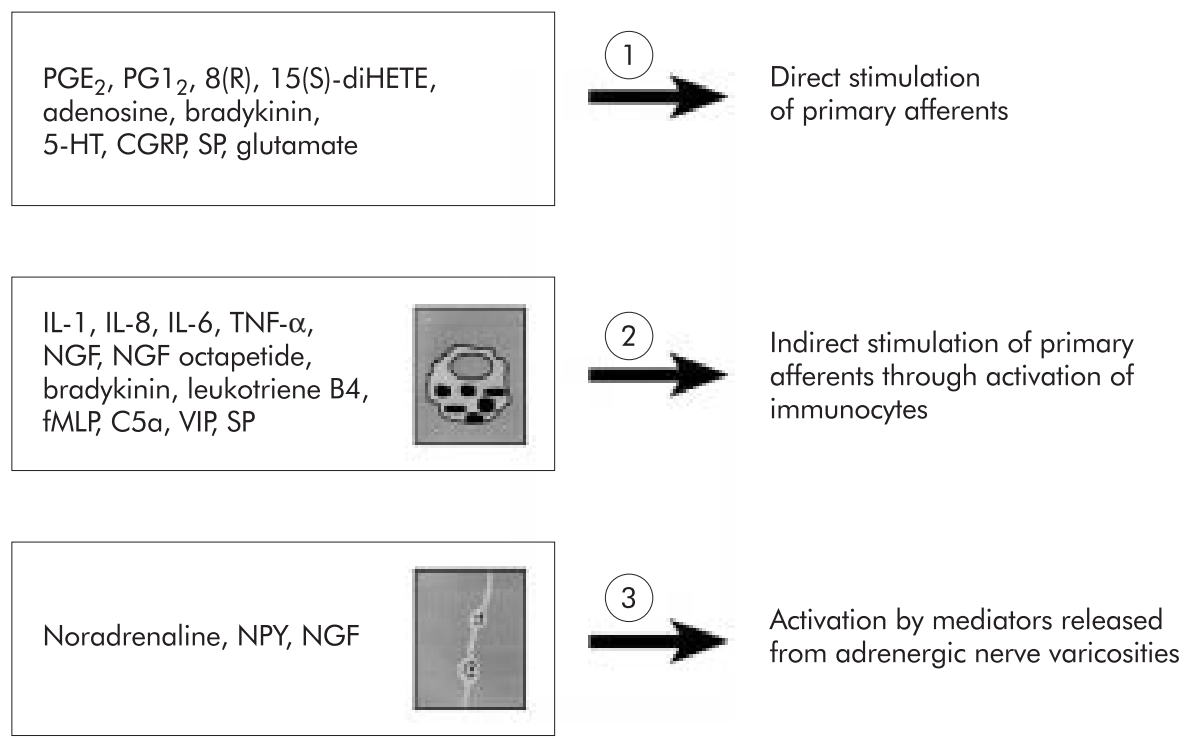

Figure 1 Substances and major local pathways involved in triggering hyperalgesia to distension within the gut. Note that several mediators such as substance $P$ (SP) may directly and indirectly influence the threshold of response of afferent fibres to a mechanical stimulus. CGRP, calcitonin gene related peptide; C5a, complement 5a; fMLP, formyl-methionyl-leucyl-phenylanine; IL, interleukin; NGF, nerve growth factor; NPY, neuropeptide $Y ; P_{2}$, prostacyclin $\mathrm{I}_{2} ; \mathrm{PGE}{ }_{2}$, prostaglandin $\mathrm{E}_{2} ; \mathrm{TNF}$, tumour necrosis factor; $\mathrm{VIP}$, vasoactive intestinal peptide; 5-HT, serotonin; 8(R), 15(S)-diHETE, 8(R), 15(S)-dihydroxyeicosatetraenoic acid. Modified from Coelho and colleagues. ${ }^{34}$

cascade is characteristic of inflamed tissues and is thought to be responsible for persistent pain triggered by local inflammation as acute administration of these substances triggers a transient algesic state in animals.

Some of the candidate proalgesic mediators released by local structures during inflammation appear to play a more prominent role in the genesis of intestinal hyperresponsiveness to mechanical stimuli. 5-HT is known to activate primary afferents. Studies on pseudoaffective (cardiovascular reflex) responses to intestinal distension suggest that its action is mediated through a $5-\mathrm{HT}_{3}$ receptor subtype coupled to a sodium channel on primary afferent endings. Accordingly, intravenous administration of low doses of $5-\mathrm{HT}_{3}$ antagonists have been shown to have potent visceral analgesic activity in response to duodenal distension in rats. ${ }^{5}$ These findings have been confirmed in numerous studies using different animal models of visceral pain. It is probable however that other 5-HT receptor subtypes, such as $5 \mathrm{HT}_{1 \mathrm{~A}}$ receptors, are also involved in mediation of visceral nociceptive input. ${ }^{67}$

Bradykinin receptors are localised on nociceptive sensory neurones. This mediator plays a major role in a variety of inflammatory processes ${ }^{8}$ and is involved in the mediation of pain and hyperalgesia caused by irritant substances in many animal models. Bradykinin receptors are subdivided into two subtypes. The $\mathrm{B}_{2}$ subtype is responsible for mediating most of the known physiological and pathophysiological actions of the kinins. There is however increasing evidence to suggest that $\mathrm{B}_{1}$ receptors, which bind with higher affinity than $B_{2}$ receptors to the bradykinin metabolite des-Arg9-BK, may play an important role in the processes that follow certain types of tissue injury, as under these circumstances they are selectively upregulated.

The antinociceptive effects of bradykinin antagonists have already been demonstrated at visceral level. In animal studies NPC-567, a non-selective $B_{1}$ and $B_{2}$ receptor antagonist, has been shown to decrease pain induced by intraperitoneal administration of acetic acid and urate crystals. ${ }^{9}$ Suppression of carrageenan induced hyperalgesia and hyperthermia by local administration of this antagonist showed that bradykinin induced neuronal sensitisation was also involved in the carrageenan reaction. ${ }^{10}$

Adenosine is a neuromediator in many nerve structures. Receptors of the $\mathrm{A}_{2}$ subtype are coupled to sodium channels on terminal endings of visceral primary afferents but the role of adenosine in triggering nociceptive messages from visceral nerve endings remains to be explored.

Tachykinins and calcitonin gene related peptide (CGRP) may also have an important role in the transmission of nociceptive messages from the gastrointestinal tract. C afferent fibres are thought to contain "silent receptors" for neurokinins which become sensitised by inflammatory processes in peripheral tissues. A large body of experimental findings also supports the involvement of SP and its receptors in pain transmission.

\section{LONG TERM EFFECT ON SENSORY PATHWAYS}

Nerve remodelling occurring during inflammation can trigger chronic hypersensitivity in the submucosa and other intestinal structures. ${ }^{11}$ These changes are complex, time dependent, and related to the nature of inflammation. The acute phase of Nippostrongylus brasiliensis infection is associated with a 2.5 -fold increase in nerve content of the tissues, chiefly as a result of axonal dilatation. During the recovery phase (14-28 days later) when mast cell hyperplasia persists, the mean cross sectional area of the nerves decreases but there is an increase in the diameter of small fibres. This observation is consistent with the idea that nerve regeneration changes sensitivity. Additional data in rats suggest that mucosal nerves, particularly B-50 immunoreactive nerves, are in a constant state of modelling. ${ }^{12}$

It has been known for many years that afferent fibres that have been injured as a result of tissue damage become more sensitive to mechanical, chemical, and probably thermal stimuli. ${ }^{13}$ More information is now available concerning the nature of the mediators involved in this response, particularly those which interact at polymodal nociceptors.

Increased sensitivity at the level of the sensory neurones, presumably at their peripheral endings, is not the only way by which mediators can enhance sensitivity. Other mechanisms which promote hypersensitivity include direct receptor induced opening of calcium and sodium channels, upregulation or downregulation of receptors on nerve endings ( triggered by changes in the number and proximity of resident immunocytes), and changes in the size or distribution of sensory neuronal endings. ${ }^{2}$ 


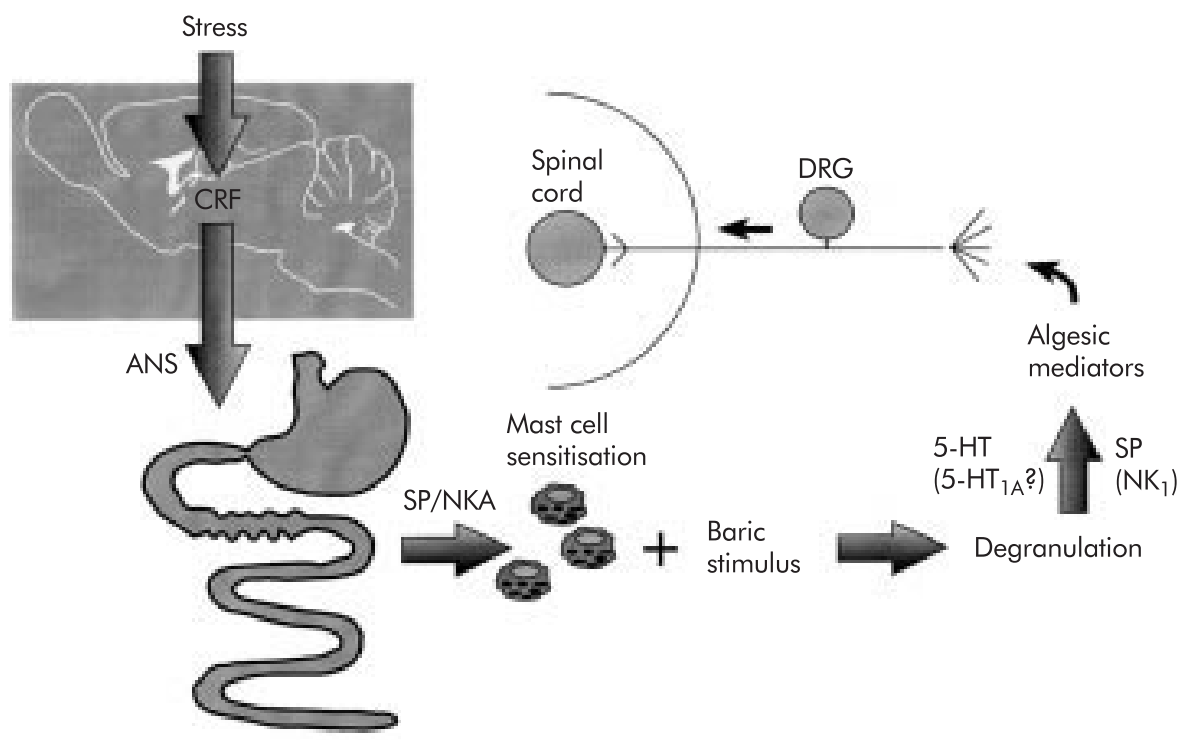

Figure 2 Pathways, structures, and mediators involved in stress induced hyperalgesia to visceral (rectal) mechanical stimulus. ANS, autonomic nervous system; CRF, corticotrophin releasing factor; DRG, dorsal root ganglia; NKA, neurokinin A; SP, substance P; 5-HT, serotonin.

Peripheral injury of primary afferent sensory neurones and permanent activation from directly or indirectly released local algesic mediators are both known to increase excitability of the dorsal horn. The role of peripheral injury in maintaining a hyperexcitable state is important as it facilitates amplification of nociceptive inputs (the so called "wind up" phenomenon) which may persist for hours or days after the peripheral noxious stimulus has disappeared. This form of hyperexcitability may play an important role in the pathogenesis of hyperalgesia.

It has also being postulated that injury evokes action potentials that are conducted antidromically along branches of nociceptor axons, or other terminal endings, where they cause release of pain provoking chemical mediators which sensitise other nociceptors in the same proximity. Until now, evidence of cross sensitisation between visceral and somatic afferents has been lacking, even though both are known to project spinally on the same interneurones. ${ }^{14}$ There is now evidence that visceral inflammation affects sensory inputs from the inflamed viscera as well as from noxious stimuli originating on the surface of the skin. In a cat model of urinary bladder inflammation, sensitisation at the level of the spinal cord was shown to selectively increase the intensity and number of ascending postsynaptic messages. ${ }^{15}$

Interestingly, in Crohn's disease only $40 \%$ of patients exhibit rectal hypersensitivity which suggests that the "wind up" phenomenon is not constant for visceral pain or that it is counterbalanced by activation of descending bulbospinal inhibitory pathways, a possibility that is supported by the fact that somatic hypoalgesia frequently accompanies the condition. $^{16}$

\section{EXPERIMENTAL MODELS OF INFLAMMATION INDUCED HYPERSENSITIVITY}

Intraperitoneal injection of acetic acid to rats triggers abdominal contractions as a manifestation of pain. Tachykinin antagonists, such as SR 48968, which act on the neurokinin $\mathrm{NK}_{2}$ receptor subtype, selectively reduce abdominal cramps while antagonists at the $\mathrm{NK}_{1}$ receptor are inactive. ${ }^{17}$ Abdominal ileus following surgery is also reduced by $\mathrm{NK}_{2}$ but not by $\mathrm{NK}_{1}$ antagonists. These observations suggest that nociceptive messages from the inflamed peritoneum involve neurokinin A (NKA) rather than SP as a mediator.
Systemic infusion of selective $\mathrm{NK}_{1}$ (GR 73632) or $\mathrm{NK}_{2}$ (GR 64349 ) receptor agonists have been shown to have different effects during graded rectal distension in non-inflamed rectum. $\mathrm{NK}_{1}$ stimulation selectively enhances the rectocolonic inhibitory reflex while $\mathrm{NK}_{2}$ stimulation selectively increases the frequency of abdominal cramps. ${ }^{18}$ The same selectivity of effect was observed with antagonists directed to $\mathrm{NK}_{1}$ (CP 96345, RP 67580) and $\mathrm{NK}_{2}$ (SR 48968, MEN 10376) receptors. Thus $\mathrm{NK}_{1}$ antagonism reversed colonic inhibition in response to rectal distension without affecting the abdominal response whereas $\mathrm{NK}_{2}$ antagonism selectively reduced visceral pain and reduced the frequency of abdominal contractions. ${ }^{19}$ The involvement of SP in visceral hyperalgesia related to intestinal inflammation has been demonstrated by the positive correlation between colonic inflammation with abdominal pain and increased concentrations of SP in chemically induced colitis. ${ }^{20}$

CGRP is present in a large number of splanchnic afferents. Its immunoreactivity largely disappears from the gastrointestinal tract after splanchnic section or treatment with the sensory neurotoxin capsaicin. ${ }^{21}$ Approximately 50\% of CGRP immunoreactive afferent neurones also show SP or NKA immunoreactivity. CGRP released from peripheral terminals of primary afferents is thought to play an important role in the development of visceral hyperalgesia. It is also possible that sensory inputs are modified by other peripherally released peptides which cause changes in blood flow, smooth muscle contractions, immune reactivity, and/or mast cell degranulation.

Intracerebroventricular injection of CGRP in mice inhibits the nociceptive response in the writhing test. ${ }^{22}$ Intravenous administration of the CGRP ${ }_{1}$ receptor antagonist (h-CGRP ${ }_{8-37}$ ) has been shown to suppress abdominal cramps induced by intraperitoneal administration of acetic acid in conscious rats. ${ }^{23}$ Unlike the selective $\mathrm{NK}_{2}$ antagonists, CGRP antagonism also blocks inhibition of gastric emptying in animals with peritonitis. Intravenous administration of CGRP itself mimics the influence of acetic acid on gastric and abdominal responses. It has also been demonstrated that CGRP is involved in the mediation of pain in response to lower intestinal distension. In accordance with these findings, the CGRP antagonist h-CGRP ${ }_{8-37}$ has been shown to reverse the sensitising effects (allodynia) of acetic acid on nociceptive response to colorectal distension following intracolonic administration of acetic acid to rats. ${ }^{23}$ 
Mast cell degranulation induced by administration of $\mathrm{BrX}$ 537 induces rectal allodynia in rats 4-6 hours after administration. Its mechanism of action involves 5-HT, but not histamine, mainly via an action on $5-\mathrm{HT}_{1 \mathrm{~A}}$ receptors. ${ }^{24}$ Intraperitoneal administration of endotoxin (lipopolysaccharide from Escherichia coli) delays biphasic rectal allodynia which accompanies mast cell degranulation and cytokine (IL-1 $\beta$ and tumour necrosis factor $\alpha($ TNF- $\alpha)$ ) release from the CNS. A process of degranulation is implicated because allodynia is suppressed by doxantrazole (a mast cell stabiliser) and also by intracerebroventricular administration of an IL-l receptor antagonist, a selective inhibitor of IL-1 converting enzyme, or a soluble form of the TNF- $\alpha$ receptor (fig 2). Vagal fibres also play an important role in limiting centrally mediated hyperalgesia as the lipopolysaccharide induced hyperalgesic state is enhanced after vagus nerve treatment with capsaicin. ${ }^{25}$

$N$ brasiliensis produces long lasting (1-3 months) visceral hyperalgesia in laboratory animals which is shown by exacerbation of the hypotensive response to intestinal distension. ${ }^{26}$ This effect is limited to intestinal segments where there is an increase in the number of mucosal mast cells which release tachykinin as the response is attenuated by selective $\mathrm{NK}_{1}$ as well as $\mathrm{NK}_{2}$ selective antagonists. Bradykinin and its major metabolite Des-Arg9-BK are also involved in this mechanism and administration of either substance selectively blocks the increase in reactivity without affecting the basal sensitivity. ${ }^{27}$

Repeated oral administration of low doses of diquat, a herbicide present as food residue, induces a mild gastrointestinal inflammation associated with an increased sensitivity to distension in rats. This long term effect is temporarily blocked by $\mathrm{NK}_{2}$ receptor antagonists suggesting that NKA is also involved in visceral hypersensitivity. ${ }^{28}$

\section{NON-INFLAMMATORY MEDIATORS OF VISCERAL PERCEPTION}

Stress has been shown to alter perception of colonic or rectal distension in humans, particularly in patients with IBS. ${ }^{29}$ In rats, partial restraint stress is associated with a transient hyperalgesia to distension, more so in female than in male animals. ${ }^{30}$ The finding that this effect is blocked by doxantrazole, a mast cell stabiliser, suggests that mast cell degranulation is involved in the mechanism. Stress is thought to increase the synthesis of mast cell products such as histamine $^{31}$ and stress sensitised mast cells have a greater ability to degranulate under a visceral baric stimulus than unsensitised mast cells (unpublished data). This form of stress induced rectocolonic hyperalgesia however is not associated with a significant increase in colonic myeloperoxidase activity.

Intracolonic infusion of glycerol has been shown to induce spontaneous abdominal cramps accompanied by increased responsiveness to colonic distension. ${ }^{32} 5-\mathrm{HT}_{3}$ receptor antagonists and indomethacin both suppress the abdominal cramps triggered by glycerol. The $5-\mathrm{HT}_{3}$ antagonists are more active when injected intracolonically than when administered by other routes suggesting a local site of action.

Local infusion of glutamate at doses that are inactive when injected intraperitoneally have also been shown to produce abdominal cramps. This response occurs without histological, biochemical, or immunological evidence of an inflammatory reaction, and is not induced by general muscular hypertonicity as concomitant recording of other somatic striated muscle cells is unaffected by intracolonic administration of glutamate. Thus the mechanism of action of glutamate remains unclear.

Proteinase activated type 2 receptors, expressed by primary afferent neurones, have been described at the periphery. ${ }^{33}$ These neurones are activated by tryptase derived from mast cells, as well as by trypsin. A tethered peptide (SLIGRL), cleaved as a result of tryptase or trypsin, activates the receptor. Intracolonic infusion of this activating receptor (PAR-2-AP) was shown to delay rectal allodynia, an effect that was not observed after systemic administration. ${ }^{34}$ The response was not associated with increased tissue myeloperoxidase activity suggesting that the process dose not involve intestinal inflammation.

\section{CONCLUSIONS}

Even though our knowledge of the importance and site of action of the mediators responsible for gastrointestinal hyperalgesia has improved considerably, the exact role played by these mediators in the pathophysiology of IBS in humans remains unclear. Despite this, it is likely that with continued research several candidate drugs will be developed in the years to come. ${ }^{35}$

Pain sensation in animal models appears to be triggered by rectal distension. Enhancement of the nociceptive response by immunological and non-immunological stimuli has been widely evaluated but few studies have investigated spontaneous nociception generated in pathophysiological situations.

Both inflammatory and non-inflammatory mediators participate in the genesis of visceral hyperalgesia in humans. Similar neuropeptides appear to be released by immunocytes, and intrinsic and extrinsic nerves. They exert their effect by binding to receptors located on sensory nerve endings.

Stress and an excess of certain mediators have the potential to trigger a form of visceral hyperalgesia which is not associated with the classic model of intestinal inflammation.

\section{Authors' affiliations}

L Bueno, J Fioramonti, Department of Neurogastroenterology and Nutrition, Institut National de la Recherche Agronomique,

Neurogastroenterology Unit INRA, 180 Chemin de Tournefeville, BP3

31931 Toulouse Cedex 9, France

\section{REFERENCES}

1 Purcell WM, Atterwill CK. Mast cells in neuroimmune function: neurotoxicological and neuropharmacological perspectives. Neurochem Res 1995;20:521-32.

2 Kennedy C, Leff P. Painful connection for ATP. Nature 1995;377:385-6.

3 Tracey DJ, Walker JS. Pain due to nerve damage: are inflammatory mediators involved? Inflamm Res 1995:44:407-11.

4 Stead RH, Kosecka-Janiszewska U, Oestreicher AB, et al. Remodelling of B-50(GAP-43)- and NSE-immunoreactive mucosal nerves in the intestines of rats infected with Nippostrongylus brasiliensis. J Neurosci $1991 ; 11: 3809-21$

5 Moss HE, Sanger GJ. The effects of granisetron, ICS 205-930 and ondansetron on the visceral pain reflex induced by duodenal distension. Br J Pharmacol 1990;100:497-501.

6 Murphy AZ, Murphy RM, Zemlan FP. Role of spinal serotonin receptor $_{1}$ subtypes in thermally and mechanically elicited nociceptive reflexes. Psychopharmacology 1992;108:123-30.

7 Zemlan FP, Murphy AZ, Behbehani MM. 5- $\mathrm{HT}_{1 \mathrm{~A}}$ receptors mediate the effect of the bulbospinal serotonin system on spinal dorsal horn nociceptive neurones. Pharmacology 1994;48:1-10.

8 Barthon JM, Proud D. Bradykinin antagonists. Annu Rev Toxicol 1991:31:129-62.

9 Steranka LR, Manning DC, Dehaas CJ, et al. Bradykinin as a pain mediator: receptors are localized to sensory neurones and antagonists have analgesic actions. Proc Natl Acad Sci USA 1988;85:3245-52.

10 Costello AH, Hargreaves KM. Suppression of carrageenan-induced hyperalgesia, hyperthermia and edema by a bradykinin antagonist. Eur J Pharmacol 1989;171:259-63.

11 Sharkey KA, Coggins PJ, Tetzlaff W, et al. Distribution of growth-associated protein, B-50 (GAP-43) in the mammalian enteric nervous system. Neuroscience 1990;38:13-20.

12 Devor $\mathbf{M}$. The pathophysiology of damaged peripheral nerves. In: Wall PD, Melzack R, eds. Textbook of pain, 3rd edn. Edinburgh: Churchill Livingstone, 1994:79-100.

13 Mayer EA, Gebhart GF. Basic and clinical aspects of visceral hyperalgesia. Gastroenterology 1994;107:271-93.

14 Carstens E. Neural mechanisms of hyperalgesia: peripheral or central sensitization? NIPS 1995;10:260-5.

15 McMahon SB. Mechanisms of sympathetic pain. Br Med Bull 1991;47:584-600.

16 Bernstein CN, Robert ME, Kodner A, et al. Is there an irritable bowel syndrome (IBS) component in patients with ileal Crohn's disease (CD)? Gastroenterology 1993;104:A477. 
17 Julia V, Buéno L. Tachykininergic mediation of viscerosensitive responses to acute inflammation in rats: evidence for a CGRP-induced release of tachykinins. Am J Physiol 1997;272:G141-6.

18 Julia V, Morteau O, Buéno L. Involvement of $\mathrm{NK}_{1}$ and $\mathrm{NK}_{2}$ receptors in viscerosensitive responses to rectal distension. Gastroenterology 1994; 107:94-102

19 Birch PJ, Harrisson SM, Hayes AG, et al. The non-peptide NK, receptor antagonist $( \pm)$ CP 96345 produces antinociceptive and anti-oedema effects in the rat. Br J Pharmacol 1992;105:508-10.

20 Kishimoto S, Kobayash $\mathrm{H}$, Machino $\mathrm{H}$, et al. High concentrations of substance $\mathrm{P}$ as a possible transmission of abdominal pain in rats with chemical induced ulcerative colitis. Biomed Res 1994;15:133-40.

21 Sternini C. Enteric and visceral afferent CGRP neurones. Targets of innervation and differential expression patterns. Ann NY Acad Sci 1992;657:170-86.

22 Delree $\mathbf{P}$, Martin D, Sadzot-Delvaux $C$, et al. In vitro and in vivo modulation of 5-hydroxytryptamine-, thyrotropin-releasing hormone- and calcitonin-gene related peptide-like immunoreactivities in adult rat sensory neurones. Neuroscience 1992:51:401-10.

23 Plourde V, Boivin M, St-Pierre S, et al. CGRP plays a role in mediating visceral nociception induced by rectocolonic distension in the rat. Gastroenterology 1995;108:A669.

24 Coelho AM, Fioramonti J, Bueno L. Mast cell degranulation induces delayed rectal allodynia in rats: role of histamine and 5-HT. Dig Dis Sci 1998;43:727-37.

25 Coelho AM, Fioramonti J, Bueno L. Systemic lipopolysaccharide influences rectal sensitivity in rats: role of mast cells, cytokines, and vagus nerve. Am J Physiol Gastrointest Liver Physiol 2000;279:G781-90.

26 McLean PG, Picard C, Garcia-Villar R, et al. Effects of nematode infection on sensitivity to intestinal distension: role of tachykinin NK2 receptors. Eur J Pharmacol 1997;337:279-82.
27 McLean PG, Picard C, Garcia-Villar R, et al. Role of kinin B1 and B2 receptors and mast cells in post intestinal infection-induced hypersensitivity to distension. Neurogastroenterol Motil 1998; 10:499-508.

28 Anton PM, Theodorou V, Fioramonti J, et al. Chronic low level administration of diquat increases the nociceptive response to gastric distension in rats: Role of mast cell and tachykinin receptor activation. Pain 2001;92:219-227.

29 Delvaux M. Stress and visceral perception. Can J Gastroenterol 1999;13:32-6A.

30 Gue M, Del Rio-Lacheze C, Eutamene H, et al. Stress-induced visceral hypersensitivity to rectal distension in rats: role of CRF and mast cells. Neurogastroenterol Motil 1997;9:271-9.

31 Bradesi S, Eutamene $\mathrm{H}$, Theodorou $\mathrm{V}$, et al. Effect of ovarian hormones on intestinal mast cell reactivity to substance P. Life Sci 2001;68: 1047-56

32 Botella A, Fioramonti J, Eeckhout C, et al. Intracolonic glycerol induces abdominal contractions in rats: role of 5 -HT3 receptors. Fundam Clin Pharmacol 1998;2:619-23.

33 Ricciardolo FL, Steinhoff $M$, Amadesi S, et al. Presence and bronchomotor activity of protease-activated receptor-2 in guinea pig airways. Am J Respir Crit Care Med 2000;61:1672-80.

34 Coelho AM, Vergnolle N, Wallace J, et al. Proteinase activated receptor-2 (PAR-2) activation prodices delayed rectal hyperalgesia in awake rats. Neurogastroenterol Motil 2000;14:381.

35 Bueno L, Fioramonti J, Garcia-Villar R. Pathobiology of visceral pain: molecular mechanisms and therapeutic implications. III. Visceral afferent pathways: a source of new therapeutic targets for abdominal pain. Am J Physiol 2000;278:G670-6. 